\title{
Risk and choice in childbirth: Problems of evidence and ethics?
}

\section{Muireann Quigley, Associate Editor}

What ought to be the limits to maternal autonomy and choice when it comes to childbirth? And what risks are ethically acceptable, given that the life and health of both the baby and mother are sometimes at stake? These questions run through a number of articles in this month's issue. These articles all note that maternal choice regarding the place and method of childbirth has become significant in the relevant national guidelines, as well as in government policy documents. ${ }^{1}{ }^{2}$ In principle at least, there is a current focus on womencentred care, which puts maternal decision-making at the forefront. This includes decisions on whether to have a homebirth (including unassisted birth), assisted midwife-led birth, or a hospital birth and on whether or not to have an elective caesarean section.

In the first of the articles dealing with childbirth, Lachlan de Crespigny and Julian Savulescu discuss whether homebirth is ethical given some of the potential risks which attend it (see page 807). Rather than examining those instances where maternal and/or neonatal death occurs, they concentrate on the risk to the future child of other sequelae; for example, hypoxic ischaemic encephalopathy and later associated disability. They argue that, even though the risks are low overall, competent homebirth is more risky than competent hospital birth. The reasons for these increased risks often relate to the lack of prompt access to the requisite facilities and expertise should problems arise during the home-birthing process. The authors claim that 'childbirth exposes the future child to unreasonable risk of potentially life-changing disability'. When comparing the risks to failing to put a seatbelt on a child they state: 'And if one child is permanently brain damaged because he/she did not wear a seatbelt, that is one child too many'. Yet despite the analogy with child seatbelts - a legal requirement in many jurisdictions-they stop short of endorsing a legal ban on homebirths.
Jasan Dannaway and Hans Peter Dietz explore some of the reasons why some expectant mothers are opting to give birth without a healthcare professional (either doctor or midwife) in attendance (see page 817). They, thus, deal with a subset of women who may, by de Crespigny and Savulescu's reasoning, be engaged in even riskier childbirth practices (for both themselves and the future child). The authors refer to other research which suggests that those who choose unassisted birth consider giving birth to carry risks wherever it takes place and that there are also risks which attend hospital births, especially of unnecessary interventions. They highlight how previous traumatic experiences are an important factor in the decision to make such a choice. Part of the problem here may stem from the lack of information given to women about certain options. Dannaway and Dietz note that physicians tend to focus on the risks of caesarean sections rather than vaginal births, and that some women do not feel adequately informed about options such as homebirth.

Mandie Scamell's article in this issue demonstrates that, even when expectant mothers are well-educated and wellinformed about the risks, options may be foreclosed to them if there is too much focus on abstract risk calculations and the medicalisation of birth (see page 813). Scamell's piece touches on important aspects of risk and probability also present in the other two articles. In particular she uses ethnographic data to discuss how 'gloomy imagined futures' can become emphasised within midwifery discourse. Even though extreme events or bad outcomes might be rare overall, they may shape practices of members of the profession. The consequence of this is that the boundaries between health and ill-health become blurred and the choices available to women become constrained. By contrast, later in this issue Hannah Selinger's student essay underlines the potential consequences of too much (uninformed) choice in childbirth (see page 857). She outlines some risks which attend elective caesarean sections (CS), especially abnormal placental growth in subsequent pregnancies. These include the attachment of the placenta to the CS scar tissue which can lead to catastrophic bleeding during delivery.

All of these articles raise questions about the interplay between evidence and ethics when determining obstetric policy. As such, they raise challenging questions for healthcare professionals, policymakers, and moral philosophers alike. They prompt us to ask what are 'reasonable' risks to take during childbirth, especially in cases where there is a low probability of the serious bad outcome occurring. One problem in trying to answer this question is that there is not currently adequate evidence regarding the long-term outcomes regarding homebirths, or indeed unassisted births. But, even if there were, the analysis given by Scamell ought to give us pause for thought. Does the practice of some professionals tend towards risk amplification, overly emphasising the type of gloomy imagined futures that perhaps de Crespigny and Savulescu as well as Sellinger discuss in their articles? Is this done selectively, emphasising (potential) risk in some areas of childbirth but not others (e.g. vaginal births)? Is such weight given to certain low probability risks justified, especially in light of the constraints it places (almost exclusively) on women's choices regarding childbirth? Conversely, do the relevant guidelines and government policy encourage professionals to pay less attention to some risks in the name of promoting maternal autonomy?

\section{REFERENCES}

1 National Institute of Health and Care Excellence. Caesarean Section. NICE guidelines [CG132], 2011.

2 Department of Health. Maternity Matters: Choice, Access, and Continuity of Care in a Safe Service. London: HMSO, 2007. 